\title{
El cáncer de colon y recto en cuidados paliativos: una mirada desde los significados del paciente
}

\author{
Palliative Care in Colorectal Cancer: Patient's Perceived Imeaning \\ 0 cáncer de cólon e reto em cuidados paliativos: uma olhada desde \\ os significados do paciente
}

\author{
Clara Elisa Reyes Piñeros* \\ Fundación Valle del Lili,Cali, Colombia \\ Margarita Saldarriaga Cuartas* \\ Universidad Icesi, Cali, Colombia \\ Diana M. Davalos* \\ Universidad Icesi, Cali, Colombia
}

Doi: dx.doi.org/10.12804/apl33.03.2015.06

\section{Resumen}

La presente investigación tuvo como objetivo comprender el significado y la manera personal como tres pacientes que recibían atención en cuidados paliativos afrontaron el cáncer de colon y recto y su proceso de enfermedad, cuando esta se encontraba en un estado avanzado e incurable. La metodología fue cualitativa con aproximación fenomenológica. Mediante entrevistas en profundidad se exploraron las creencias y se obtuvo información de los pacientes acerca del origen de la enfermedad, las expresiones emocionales y los recursos y obstáculos para afrontarla. Los resultados permitieron comprender que el significado construido alrededor del diagnóstico está relacionado con sus experiencias pre- vias, la información que tenían acerca de su condición y la presencia o no de síntomas. Se destaca el hecho de que no percibieron la enfermedad como amenaza, que se sintieron tranquilos frente al diagnóstico y de que no tenían conciencia de la proximidad de la muerte. Las expresiones emocionales variaron desde la tristeza, la sorpresa y la angustia al momento del diagnóstico, hasta la aceptación, la confianza en Dios y en los tratamientos médicos como vía para la curación. El seguimiento de las recomendaciones médicas y la confianza en Dios se convirtieron en las principales estrategias para afrontar la enfermedad.

Palabras clave: significados; afrontamiento personal; psicooncología; cáncer de colon y recto; cuidados paliativos.

* Clara Elisa Reyes Piñeros, Equipo de Cuidados Paliativos, Clinica Fundacion Valle del Lili; Margarita Saldarriaga Cuartas, Universidad Icesi; Diana M. Davalos, Department of Social and Behavioral Sciences, School of Public Health, University of North Texas.

La correspondencia relacionada con este artículo debe ser enviada a Clara Reyes. Correo electrónico: clarae.reyesp@gmail.com

Cómo citar este artículo: Reyes, C. E., Saldarriaga, M. \& Davalos, D. M. (2015). El cáncer de colon y recto en cuidados paliativos: una mirada desde los significados del paciente. Avances en Psicología Latinoamericana, 33(3), 439-454. doi: dx.doi.org/10.12804/ap133.03.2015.06 


\section{fibstract}

This research aimed to understand the meaning and personal coping process of three end-stage colorectal cancer patients receiving palliative care. The metodology was qualitative, with a phenomenological approach. By way of in-depth interviews, we explored beliefs and obtained information from the patients about the origin of the disease, the expression of emotions and resources and obstacles for coping. Results allowed us to understand that the patient's meaning constructed around the diagnosis relates to previous experiences, knowledge of the disease, and the presence or absence of symptoms. Importantly, patients did not perceive the disease as a threat, they felt calm about the diagnosis and were not conscious of the proximity of death. Emotional expressions ranged from sadness, surprise, and distress at diagnosis, to acceptance, trust in God, trust in the medical treatments as a way of healing. Adherence to medical advice and trust in God became main strategies for coping with the disease.

Keywords: meaning; coping; psycho-oncology palliative care; colorectal cancer.

\section{Resumo}

A presente pesquisa teve como objetivo compreender o significado e a maneira pessoal como três pacientes que recebiam atenção em cuidados paliativos afrontaram o cáncer de cólon e reto e seu processo de doença, quando encontrava-se em um estado avançado e incurável. A metodologia foi qualitativa com aproximação fenomenológica. Mediante entrevistas em profundidade exploraram-se as crenças e obteve-se informação dos pacientes acerca da origem da doença, as expressões emocionais e os recursos e obstáculos para afrontá-la. Os resultados permitiram compreender que o significado construído ao redor do diagnóstico está relacionado com suas experiências prévias, a informação que tinham acerca de sua condição e a presença ou não de sintomas. Destaca-se o fato de que não perceberam a doença como ameaça, que sentiram-se tranquilos frente ao diagnóstico e de que não tinham consciência da proximidade da morte. As expressões emocionais variaram desde a tristeza, surpresa e angústia ao momento do diagnóstico, até a aceitação, confiança em Deus e nos tratamentos médicos como via para a cura. O seguimento das recomendações médicas e a confiança em Deus converteram-se nas principais estratégias para afrontar a doença.

Palavras-chave: significados; afrontamento pessoal; psico-oncologia; cáncer de cólon e reto; cuidados paliativos.

En el mundo, el cáncer de colon y de recto ocupa el tercer lugar entre los diversos tipos de cáncer (Ferlay, 2010). La prevalencia es del 9.7\% (1.23 millones de personas), y es la segunda causa de muerte por cáncer en los Estados Unidos (Labianca y Merelli, 2010). En Colombia, el Instituto Nacional de Cancerología reportó para Colombia 203907 muertes por cáncer en el 2010, equitativamente distribuidos entre mujeres y hombres (Piñeros, Pardo, Gamboa \& Hernández, 2010). En el 2009 se presentaron 312 nuevos casos de cáncer de colon y recto, y estos se convirtieron en la quinta causa de mortalidad por cáncer en Colombia. Se estima que para el año 2045 su incidencia será igual a la del cáncer gástrico en el país (Ángel, Giraldo \& Pardo, 2004).

El cáncer de colon y recto afecta al paciente en las esferas física y emocional, y a su familia. La enfermedad se asocia con sufrimiento y muerte, y con la combinación de tratamientos como la cirugía, la quimioterapia, la radioterapia y el uso de una colostomía, los cuales representan cambios en el cuerpo y alteración de la imagen corporal (Die Trill, 2007). También hay cambios en los hábitos alimenticios, el modo de vestirse, las actividades de diversión, la sexualidad y el campo laboral; esto hace que el paciente perciba la situación como una amenaza para su integridad (Martín \& Montesinos, 2010).

Adicionalmente, se han identificado cuatro factores puntuales que aumentan el riesgo de sufrimiento en el paciente: la incertidumbre o la 
falta de certeza sobre lo que sucederá en el futuro; sentimientos negativos, como miedo, tristeza o ansiedad; la sensación de pérdida de control, y la amenaza a la autoestima como consecuencia de los cambios generados por el tratamiento que alteran la autoimagen y producen la pérdida de la autonomía (Bayés, 2006).

Se sabe que la principal fuente de estrés para los pacientes con cáncer de colon y recto es la falta de control sobre la expulsión de las heces y que el estoma esté a la vista (McVey, Madill \& Fielding, 2001; Marquis, Marrel \& Jambon, 2003; Martin, Panduro, Crespillo, Rojas \& González 2010; Wu, Chau \& Twinn, 2007). Los profesionales de la salud que acompañan los procesos del paciente con esta enfermedad deben hacerlo desde una perspectiva integral que incluya cuidados médicos, psicológicos, espirituales y sociales.

Según el estadio en el cual se encuentre la enfermedad, el paciente puede curarse o pasar a un periodo de remisión. Posteriormente, si el paciente recae, llega a una fase final que se conoce como enfermedad terminal, caracterizada por ser progresiva, avanzada e incurable, con una expectativa de vida inferior a seis meses, sin opciones de tratamiento curativo, síntomas intensos, cambiantes y de origen multifactorial (Sociedad Española de Cuidados Paliativos, 2012).

En esta etapa se brindan cuidados paliativos encaminados a conservar la vida durante el proceso de aceptación de la muerte como algo inevitable. El cuidado paliativo no acelera ni pospone la muerte; se centra en proporcionar alivio del dolor integrando aspectos psicológicos, sociales y espirituales, $\mathrm{y}$ ofreciendo un sistema de apoyo para los pacientes y sus familiares (Pascual, 2003; Rojas-May, 2006; Valentín, Murillo, Valentín \& Pascual, 2003).

Las implicaciones emocionales del cáncer colorrectal en etapa terminal requieren intervenciones psicosociales que buscan la adaptación del paciente y su familia al proceso de enfermedad, a fin de lograr una sensación de vida completa y la aceptación de la muerte (Wenk, 2007). Para que estas inter- venciones tengan el impacto esperado y realmente contribuyan a favorecer la adaptación al proceso de enfermedad del paciente y sus familiares, es preciso que se ajusten a las necesidades y demandas de cada uno de los participantes. Uno de los elementos centrales es identificar los significados que cada paciente le asigna a su enfermedad, para que a partir de dichos significados se construyan intervenciones específicas y efectivas.

Un factor frecuentemente identificado en el paciente con cáncer de colon y recto, durante su proceso de afrontamiento, es el impacto negativo que les genera la ostomía, cuya presencia se acompaña de emociones como desesperación, miedo, angustia y rechazo ante los cambios que la enfermedad les impone en la forma de vestirse, alimentarse y en su sexualidad (Silva \& Shimizu, 2006; Vinaccia \& Arango, 2003).

La intervención que se llevó a cabo en los pacientes con cáncer colorrectal en etapa terminal tuvo como eje principal los significados que el paciente le asignó a su enfermedad, con un abordaje desde el construccionismo social y otras corrientes sistémicas en las que se plantea que la construcción de significados ocurre en las relaciones y en acciones coordinadas con los otros en el dominio lingüístico (el diálogo y la conversación) y tienen efectos en las actuaciones de las personas (Anderson \& Goolishian, 1988; Arcila, Mendoza, Jaramillo \& Cañón, 2010; González, Fonseca \& Jiménez, 2006).

La comprensión de los sistemas de significación es fundamental en la medida en que estos son los procesos por medio de los cuales los humanos le damos sentido a la experiencia vivida o por vivir. Tales sistemas son los ejes frente a los que se interpreta una idea o se le asigna sentido (Hernández, 2009). En esta investigación, el uso de este abordaje permitió entender que la información y las ideas sobre el cáncer colorrectal de estos pacientes fueron los referentes indispensables para que establecieran una posición frente a su enfermedad, que terminó por expresarse en emociones y comportamientos. 
La posibilidad de explorar lo que el paciente comprendía acerca de los sucesos que estaba experimentando por medio de las conversaciones, $\mathrm{y}$ mediante ellas expresar sus emociones, para integrar experiencias y emociones a los significados que el paciente tenia de sí mismo y del mundo, fue uno de los elementos centrales de esta investigación. Mediante este proceso, el terapeuta buscaba que el paciente lograra acercarse a un bienestar emocional (Anderson \& Colapinto; 1999; Gergen \& Warhus, s. f.; Maturana 1991; Tarragona, 2006).

Entendemos que los pacientes construyen sus significados y desde allí identifican recursos y obstáculos para afrontar la enfermedad. En el paciente con cáncer el proceso de afrontamiento es considerado un mecanismo que lo protege ante el estrés y le permite adaptarse a su nueva realidad (Broche \& Medina, 2011). Algunos factores importantes durante el proceso de afrontamiento son el apoyo que el paciente percibe de su familia y su círculo social, las estrategias para la resolución de problemas, la variación de sus aspiraciones, la percepción de la calidad de su vida y el manejo de los síntomas (Martín, Zanier \& García, 2003; Oliveros, Barrera, Martínez \& Pinto, 2010).

Algunas de las estrategias usadas para el afrontamiento que se observan en estos pacientes son la represión, la negación, la sustitución, la normalización y el encubrimiento. Cuando el paciente se encuentra en la fase final de su enfermedad, se confronta consigo mismo, toma conciencia de la muerte y aparecen sentimientos de desamparo, indefensión, miedo y soledad. Esto permite una reconstrucción de sus significados, valores y creencias y un cambio de sí mismo y de sus relaciones con los demás (Blair \& Cardona, 2011).

La intervención psicosocial como parte del manejo integral del paciente con cáncer de colon y recto busca crear un espacio conversacional que les permita a los participantes y al terapeuta deambular por la historia de su enfermedad desde su óptica sobre este diagnóstico y su experiencia en la convivencia con el cáncer de colon y recto.
El propósito de este estudio fue conocer los significados asignados al cáncer de colon y recto y la relación de estos con los procesos de afrontamiento en tres pacientes atendidos por el grupo de cuidados paliativos de una clínica privada. Para esto se exploró la información y las creencias de estos pacientes sobre su diagnóstico, se describieron las expresiones emocionales frente a su enfermedad y se identificaron los recursos y obstáculos para su afrontamiento.

\section{Método}

Esta investigación cualitativa tuvo como eje la subjetividad como espacio de construcción de la vida humana. Por medio de la perspectiva interpretativa y con una aproximación fenomenológica, se hizo una reflexión consciente y sistemática sobre aspectos de la vida de tres pacientes diagnosticados con cáncer de colon y recto en tratamiento paliativo, para comprender los significados asignados a su proceso. Se exploraron sus vivencias, buscando entender su realidad y describiendo el fenómeno, sin establecer relaciones causales (Quintana, 2006; Sandoval, 2002; Taylor \& Bogdan, 1990).

Para la investigación se seleccionaron, mediante muestreo intencional, tres individuos mayores de edad diagnosticados con cáncer de colon y recto que en el 2012 estaban recibiendo manejo ambulatorio de su enfermedad en el servicio de oncología y cuidados paliativos de la clínica privada en la que se realizó el estudio. La propuesta fue presentada a un grupo de pacientes y se desarrolló con quienes expresaron interés en participar. Se recolectó información mediante una ficha de datos sociodemográficos, con trece preguntas que incluían datos biográficos, estrato socioeconómico, composición familiar y ocupación. Posterior a la caracterización, se hizo una entrevista en profundidad con cada participante, la cual fue grabada en audio. En dicha entrevista se exploraron cuatro categorías de análisis: (a) creencias alrededor de la condición de enfermedad, (b) expresiones emocionales, (c) re- 
cursos para afrontar la enfermedad y (d) obstáculos para afrontar la enfermedad (tabla 1).

Tabla 1

Categorías y subcategorias

\begin{tabular}{ll}
\hline \multicolumn{1}{c}{ Categoría } & \multicolumn{1}{c}{ Subcategoría } \\
\hline $\begin{array}{l}\text { Creencias acerca de la con- } \\
\text { dición de enfermedad }\end{array}$ & Información \\
$\begin{array}{l}\text { Ideas acerca de su enferme- } \\
\text { dad, causa, pronóstico y el } \\
\text { significado que le otorgan }\end{array}$ & Explicaciones \\
$\begin{array}{l}\text { Expresiones emocionales } \\
\begin{array}{l}\text { La manera en que es expre- } \\
\text { sada la emoción }\end{array}\end{array}$ & Momento del diagnóstico \\
$\begin{array}{l}\text { Recursos para afrontar la } \\
\text { enfermedad }\end{array}$ & Recursos personales \\
$\begin{array}{l}\text { Estrategias, capacidades, } \\
\text { recuerdos y habilidades que } \\
\text { le han permitido al paciente } \\
\text { hacer frente a la enfermedad }\end{array}$ & Recursos familiares \\
$\begin{array}{l}\text { Obstáculos para afrontar } \\
\text { la enfermedad }\end{array}$ & $\begin{array}{l}\text { Obstáculos personales/ } \\
\text { familiares }\end{array}$ \\
$\begin{array}{l}\text { Aquellas situaciones físicas, } \\
\text { emocionales, cognitivas que } \\
\text { dificultan el hacer frente a la } \\
\text { enfermedad }\end{array}$ & $\begin{array}{l}\text { Obstáculos de la } \\
\text { enfermedad }\end{array}$ \\
\hline $\begin{array}{l}\text { Obstáculos sociales } \\
\end{array}$ & Recursos externos \\
\hline
\end{tabular}

Estos encuentros tuvieron una duración aproximada de una hora y se hicieron en el lugar de residencia de cada participante. El número de encuentros requeridos varió con cada participante: el primero (participante 1 [P1]) participó en una sola entrevista, el segundo participó (participante 2 [P2]) en tres entrevistas y el tercero (participante 3 [P3]) estuvo en dos entrevistas.

Las entrevistas se llevaron a cabo utilizando como referente un guion de preguntas orientadoras, se grabaron en audio y después se transcribieron. Los datos se organizaron manualmente basados en las categorías y subcategorías construidas y las emergentes. Para su validación se les pidió a los participantes verificar esta información ya cate- gorizada; una vez se tuvo un primer borrador del informe, se devolvieron los resultados a los participantes para validar la información categorizada y para hacerlos partícipes de la construcción de resultados y análisis, teniendo en cuenta que la validez depende de "la verificación con los sujetos participantes de la fidelidad con la que se sienten comprendidos y de los efectos que les ha dejado la experiencia" (Hernández, 2010,p. 113). El análisis de la información se realizó por medio de la técnica de análisis temático.

La investigación tuvo presente las consideraciones éticas relacionadas con la investigación con seres humanos, según los lineamientos de la Ley 1090 del 2006, que regula el ejercicio de la psicología en Colombia; la Resolución 008430 de 1993 del Ministerio de Salud, por medio de la cual se establecen en las normas científicas, técnicas y administrativas, para la investigación en salud, y el Manual deontológico y bioético de psicología. Como se mencionó, se devolvieron los resultados a los participantes, a fin de cumplir con el compromiso ético adquirido con ellos.

Adicionalmente, este estudio fue aprobado por el Comité de Ética Médica y Biomédica de la institución. Se obtuvo consentimiento informado de los tres participantes, en el cual se resaltó el carácter voluntario de la participación y la posibilidad de retirarse de la investigación en cualquier momento y que su participación en el estudio no tendría ninguna consecuencia para su tratamiento médico en la institución. Se mantuvo la confidencialidad de los participantes mediante el uso de seudónimos y no de sus nombres reales; a los participantes se les informó sobre los alcances del estudio y el fin académico de la información obtenida.

\section{Resultados}

A continuación se presentan los resultados de la investigación, de acuerdo con las categorías, creencias e información acerca de la condición de enfermedad, expresiones emocionales asociadas 
con el proceso de enfermedad, recursos y obstáculos para afrontarla.

\section{Categoría 1. Creencias e información acerca de la condición de enfermedad}

Esta categoría agrupó los entendimientos y saberes generales de los participantes acerca del cáncer y las creencias asociadas con las causas, el pronóstico y el significado otorgado al proceso de enfermedad.

Subcategoría 1. Información general sobre la enfermedad. La información que tenían los pacientes sobre el cáncer antes de conocer su diagnóstico era la de una enfermedad grave y, en muchos, casos mortal, que dependía especialmente del lugar de localización y del tiempo de detección. Los pacientes manifestaron: "Es una enfermedad muy agresiva según como dé y en qué parte dé” (P3). Cuando fueron diagnosticados, se plantearon la posibilidad de controlar temporalmente el cáncer, y su incurabilidad: "Es una enfermedad que uno sabe que un cáncer no se cura, se detiene mucho tiempo sí, pero no se cura y más en el caso mío que está tan avanzado; yo sé que no se cura" (P1).

Los participantes manifestaron que las consultas iniciales fueron motivadas por síntomas intensos y dolorosos en las que los médicos les dieron manejo sintomático para los cólicos, diarreas u otras molestias, sin buscar la causa de estos síntomas: "Unos cólicos tremendos que no aguantaba más" (P2), "Estaba de un lado a otro y eran cólicos; estaba una noche entera y cuando me levantaba me daba mucho daño de estómago" (P1). Posterior al diagnóstico, los participantes adquirieron información nueva que les permitió entender la posibilidad de detección temprana de la enfermedad y su estadio: "El Dr. K. me dijo que los síntomas de un cáncer en el colon se detectan muy tempranamente" (P3), "Él me dijo, no, antes te está avanzando más" (P2). El principal tratamiento fue la quimioterapia y para una de las participantes estuvo asociada al desen- lace, "Ya me tocaba hacerme la quimio porque tengo la enfermedad; entonces dije 'o me mejoro o me muero"" (P1).

Para los pacientes, el control de los síntomas, en especial la ausencia de dolor, se consideró un signo de mejoría de la enfermedad, "Yo me doy cuenta porque el dolor se me ha quitado, el dolor ya no lo tengo", "Yo pienso que el medicamento me está ayudando; está haciendo efecto" (P2). Manifestaron también que habían usado remedios caseros como tratamientos sintomáticos antes del diagnóstico o, al mismo tiempo, con la quimioterapia para atenuar sus efectos o como elementos de sanación, por recomendaciones de otros pacientes o conocidos, "Yo creo que con eso y otros remedios caseros que me estoy haciendo... Yo creo que con eso me está ayudando" (P2).

Subcategoría 2. Explicaciones sobre las causas de la enfermedad. El primer factor señalado por los tres participantes como causa del cáncer fue el hereditario: "De pronto fue porque mi papá la tenía, entonces siempre decimos que eso viene de familia" (P1). Un segundo factor fue la presencia de hábitos poco saludables como el consumo de licor y cigarrillo: "Pues yo creo, doctora, que por el asunto de, no sé si fue el trago, porque yo tomaba mucho; no sé sí fue por eso; sí, yo creo, porque yo sí tomaba mucho trago y fumaba también" (P2). E1 tercer factor mencionado en particular por uno de los participantes fue el consumo de alimentos contaminados y la posible contaminación en la empresa con elementos perjudiciales como posibles causas del cáncer: "Yo vi morir varios compañeros en la empresa, decían que posiblemente había alguna contaminación" (P3).

Subcategoría 3. Expectativas sobre el pronóstico de la enfermedad. Para los pacientes, el cáncer "terminal" significaba "muerte" y reconocían que aunque existan periodos de ausencia de enfermedad, podría haber reactivación de los síntomas "No llega un momento en que uno se 
cure en un $100 \%$, no se cura" (P3). Sin embargo, al profundizar acerca de su propia condición, dos de los participantes asociaron la ausencia de síntomas y sobre todo de dolor con la curación de la enfermedad. Al mismo tiempo, la ausencia de síntomas físicos generó expectativas de retomar las actividades previas a la aparición de la enfermedad: "El ánimo mío es después de que pase la enfermedad, es ponerme a trabajar" (P2).

La visión de un pronóstico favorable y un estado de mejoría en uno de los participantes se manifestó a través de la fe en Dios: "Sí, yo creo en Él, porqué Él es el que me está curando; yo ya me estoy sanando y es por Dios, y claro, los médicos también, pero más es Dios" (P2), sin desconocer que los tratamientos médicos (quimioterapia) pueden llegar a ser una cura de la enfermedad: "No, ya pienso ya distinto, ya digo que ya prácticamente me curé; en estos momentos digo que ya me curé" (P2). Adicionalmente, los participantes no conocían el significado del programa de cuidados paliativos, pero hicieron referencia al buen servicio y apoyo que recibieron en esa consulta.

\section{Categoría 2. Expresiones emocionales del paciente}

Esta categoría abordó las expresiones emocionales de los pacientes asociadas al momento del diagnóstico y al momento de la entrevista. Ante el diagnóstico expresaron que habían sentido "desesperación"; describieron que los fuertes dolores, las múltiples visitas a urgencias y la asistencia a diferentes consultas convirtieron este periodo en el momento de mayor tensión: "El peor momento fue cuando sentí los síntomas" (P3). La intensidad de los síntomas se relacionó con preocupación y sufrimiento. Uno de los participantes recuerda esos días como difíciles para él y su familia: "Estaba desesperado, no veía una solución a lo que me pasaba, sentía que era demasiado para mí; fueron unos días muy difíciles para todos" (P3).
Ante la confirmación del diagnóstico refirieron sentir "sorpresa" y "tristeza", asociaron lo que sentían físicamente con la información que tenían sobre el cáncer: "En ese momento yo me sentía ya muy mal, con el diagnóstico, yo pensé que ese era ya el final, sentí que todo se venía abajo" (P3). El resultado de la biopsia causó preocupación: "Fue más de una hora hasta llegar a la casa, estaba preocupado, pensaba y pensaba en lo mismo, todo lo que me había dicho la médica" (P3), y hasta el momento en que se realizó la intervención quirúrgica manifestaron sentirse "desesperados", además de sentir "rabia" y "tristeza". "No me hacían nada para la enfermedad, yo la sentía ahí y fue horrible esperar" (P3).

Posterior al diagnóstico, cuando ocurrieron las entrevistas de esta investigación, los pacientes estaban en tratamiento y expresaron sentirse tranquilos, en calma: "Bien, gracias a Dios, bien, estaba tranquilo" (P2). "Me sentí tranquilo cuando me dijeron lo que tenía, por lo menos ya se iba a hacer algo" (P3). La mejoría de los síntomas fue factor de bienestar y "alegría", por estar recibiendo los tratamientos necesarios para su enfermedad: "Yo me siento muy contento, vale la pena aguantarse las maluqueras, mantengo de buen ánimo" (P2). Sin embargo, también expresaron "intranquilidad" por la posibilidad de progresión de la enfermedad y uno de los participantes describió la sensación de no tener interés en nada: "He estado intranquilo porque creo y he escuchado que la enfermedad solo se controla, no se cura"; "Soy una persona que no tiene interés de nada" (P3).

En dos pacientes, la experiencia con la colostomía estuvo acompañada de emociones negativas, fue motivo de "incomodidad", "vergüenza" e inhibición social; además de generar "miedo" a establecer intimidad en la relación de pareja: "Uno se va aburriendo porque se ve uno así (señala la colostomía)"; "Sí, ya no soy el mismo, y eso me aburre, ahora no me provoca salir a pasear y conocer gente" (P2). 


\section{Categoría 3. Recursos personales, familiares y externos para afrontar la enfermedad}

Subcategoría 1. Recursos personales. Las acciones de los pacientes por comprender su diagnóstico incluyeron la solicitud de conocer la verdad de su situación a sus médicos tratantes y el poder tener un adecuado control de los síntomas, principalmente del dolor. "Yo le dije al médico: 'doctor, dígame la verdad"”, "Por favor le pido que yo no vaya a sufrir que no vaya a sentir mucho dolor" (P3). La información recibida les permitió conocer la temporalidad de los efectos secundarios de la quimioterapia: "Yo me hago la quimioterapia y estoy enferma una semana, dos semanas y yo sé que de allí me levanto" (P2). La aceptación de los acontecimientos y el tener una actitud positiva frente a la enfermedad fueron también elementos importantes: "Yo pienso que lo positivo que yo tengo, vamos para delante, yo pienso que yo con esto no me voy echar a la muerte, a mí esto me ha ayudado mucho porque a mí me mandan una pasta y yo me la tomo con fe" (P1). Expresaron una recomendación a quien estuviese viviendo lo mismo que ellos: "Que sea fuerte y que le haga frente a la enfermedad. Si la persona se somete y desea morirse eso no ayuda" (P3).

Los tres participantes resaltaron la importancia de la fe en Dios y reconocieron su acercamiento a la religión a partir de la enfermedad, incluyendo las oraciones como forma de ayuda a los tratamientos en su labor sanadora: "Yo le pedí a mi Dios que saliera bien", "Mi Dios, el único que nos sana es mi Dios, es el único" (P2).

Subcategoría 2. Recursos familiares. El cónyuge y la familia extensa fueron considerados fuente de cuidados. Uno de los participantes reconoció el apoyo recibido de su esposa, su compañía permanente durante el proceso hospitalario, los cuidados proporcionados, la recursividad para ahorrar en los insumos para la colostomía y sus palabras: "Ella duró desde ese día que a mí me internaron en la clínica 22 días que no fue a la casa", "Ella era la que me limpiaba y yo no me daba cuenta", "Confiemos en Dios que todo va salir bien... Me dio tranquilidad sus palabras" (P3). En otro caso, miembros de la familia extensa buscaron alternativas para agilizar el diagnóstico y tratamiento, “Tío, a esto hay que ponerle remedio, vamos a ponerle remedio, vamos pa' donde el médico a ver qué le dice el médico" (P2). Los familiares acompañaron a los pacientes a las citas médicas, hospitalizaciones, y aplicación de quimioterapia; así mismo, ofrecieron los cuidados básicos relacionados con la alimentación, la ropa, recursos económicos y apoyo para realizar trámites administrativos ante la entidad de salud: "Cuando estoy enferma, pues ellas cocinan y me dan", "Mis hermanos dijeron: 'nosotros vamos a pagarle el seguro"" (P1).

Subcategoría 3. Recursos externos. Los recursos externos utilizados por los participantes antes del diagnóstico fueron diferentes tipos de medicina alternativa, en búsqueda de alivio para los síntomas "Un médico que veía las orinas por allá en Pradera", "Donde un señor que me dio unas agüitas; él me dijo tienes problemas del colon" (P3). Durante el tratamiento, recibieron algunas recomendaciones de los compañeros en la sala de quimioterapia y de otras personas, acerca de alimentos o remedios caseros que les podían ayudar dentro de los que se contaron: la sábila, el bicarbonato de soda, plantas medicinales (anamú, caléndula), la guanábana, el limón y el gano café, entre otros: "Cuando iba a la quimioterapia cogía un cristal de sábila y lo licuaba con jugo de naranja o limón y me tomaba eso", "Me dijeron que la guanábana, que consumiera guanábana permanentemente" (P3).

Ante el tratamiento quirúrgico un paciente señaló que la educación recibida, la información del cirujano tratante, los medicamentos para controlar la angustia y el ambiente calmado de una habitación individual fueron importantes en su proceso: "Que la droga ayuda un poco, y la tranquilidad (refiriéndose a la habitación)" (P3). El apoyo recibido por 
el grupo de cuidados paliativos fue importante; los pacientes reconocieron la presencia del médico y la psicóloga en las intervenciones "Me ayudó muchísimo la cita que he tenido más de un año con cuidados paliativos", "Que las personas que se recuperan del cáncer parte de lo que los ayuda, son las personas que lo rodean, tanto los médicos, como el psicólogo dándole aquellas palabras de aliento" (P3).

Las oraciones de otras personas, familiares, amigos fueron parte de las cosas que les ayudaron. "Yo tengo un cáncer terminal y confío que ustedes oren por mí y me ayuden en algo", "Venían muchos amigos aquí, amigos míos, un vecino de aquí, dueño de la casa de aquí y rezaban" (P3).

\section{Categoría 4. Obstáculos personales, familiares, de la enfermedad y sociales para afrontar la enfermedad}

Subcategoría 1. Obstáculos personales y familiares. Uno de los participantes refirió que lo que pensaba sobre la enfermedad, aparte de afectarlo emocionalmente, no le permite proyectarse a futuro, no hacía planes con su esposa y sentía limitaciones para relacionarse con otras personas: "Los pensamientos míos murieron ahí; yo digo que tuve antes de la enfermedad, tenía muchos planes", "Ahora mis pensamientos no se extienden hasta allá" (P3).

Existen también emociones negativas que los participantes perciben de sus familiares: "El dolor más grande fue cuando nos dijeron que su papá ya no va vivir", "Ella sufrió mucho durante un año con mi colostomía" (P3). Una paciente refiere el sufrimiento de sus hermanas ante su quimioterapia y los efectos secundarios: "Para qué me tienen que hacer todo eso que me hacen (quimioterapia), les da tristeza ver todo lo que me hacen, dicen: ¿por qué mi propia hermana? Ellas dicen: 'usted que ha sido tan buena"" (P1).

Subcategoría 2. Obstáculos de la enfermedad. El principal obstáculo de la enfermedad en estos pacientes fue la colostomía y las limitacio- nes que esta les generó, entre ellas las actividades básicas de cuidado personal, vestirse, bañarse "Incómodo, por lo menos para ponerme las medias, para ponerme el zapato por este lado" (P2). Socialmente, hubo comportamientos de inhibición, como evitar salir a compartir con amigos por temor a que la bolsa se desprenda, al sonido de los gases; además, manifestaron que no pueden participar en actividades en el agua, ni hacer ejercicio físico o de velocidad "Esto debo cambiarlo, aquí al baño tengo que ir cada hora, cada dos horas máximo", "A mí me ven por aquí todavía, la bolsa colgando y se impresiona la gente" (P3). Han suspendido las relaciones sexuales: "Ya después de que me cierren esto y todo termine, vuelvo a buscar a las mujeres" (P2).

Adicionalmente, refirieron como obstáculo el cambio de roles en la familia, los gastos que generó la enfermedad por los desplazamientos a los tratamientos y la alimentación que debía recibir: "Yo no pensaba en lo de mi enfermedad, sino en la situación económica, porque una enfermedad de estas, da mucho gasto" (P1). El no poder hacer esfuerzos físicos los limitó para actividades laborales y del campo: "Yo siempre he sido trabajador en el campo ya no puedo echar pala, ahora tengo que estarme en la casa" (P2).

Subcategoría 3. Obstáculos sociales. El sistema de seguridad social colombiano fue visto como un obstáculo en las diferentes etapas del proceso: prediagnóstico, diagnóstico, cirugía, entrega de insumos para la colostomía y pago de incapacidades.

Antes del diagnóstico, se manejaron solamente los síntomas, no se hicieron pruebas diagnósticas oportunamente y como resultado se hizo un diagnóstico tardío: "El médico no hacía sino mirar y tratarme con unas pastas, pero no le mandaban a uno los exámenes; a lo último me tocó pagar particularmente para que me atendieran" (P2). Al momento del diagnóstico, la empresa promotora de salud (EPS) se demoró en autorizar la cirugía de resección del tumor: "La aprobación de la 
operación en la SOS se demoró como siete meses" (P3), y posteriormente no le autorizaron los insumos para su colostomía "La SOS no me daba todos los implementos para eso... Yo me gastaba mensualmente casi 2.000.000" (P3).

\section{Discusión}

Los resultados de nuestra investigación reflejan la experiencia de trabajo con los participantes y la evidencia encontrada en la literatura. Por medio de los relatos acerca de su proceso de enfermedad; sus creencias; información, entendimiento y explicaciones de las causas del cáncer; sus emociones al momento del diagnóstico y de las entrevistas, y los recursos y obstáculos que identificaron para contribuir o impedir el proceso de afrontamiento, evidenciamos cinco hallazgos que merecen ser discutidos.

Primero, el diagnóstico de los participantes se hizo en una fase avanzada de la enfermedad debido a la dificultad para llegar a la consulta de un especialista. Inicialmente, los pacientes consultaron al médico general de la institución prestadora de salud, quien, según su criterio y la regulación del sistema de salud colombiano, remitió al médico especialista. Pese a que para ese momento los tiempos de espera estipulados eran de veinte días para la consulta especializada y de treinta días para la cirugía programada (Supersalud, 2009), en nuestro estudio la consulta con el especialista tuvo un tiempo de espera de seis a ocho semanas, y la cirugía de resección del tumor aproximadamente, siete meses. Se evidenció una falla en el sistema, con un diagnóstico tardío de la enfermedad y un impacto negativo en su pronóstico. Hay una ausencia de la perspectiva preventiva y un enfoque en las intervenciones correctivas. Si bien esto no puede generalizarse a toda la población, sí debe despertar reflexiones en torno a la necesidad de generar intervenciones en dos niveles: la detección temprana del cáncer de colon y recto, que puede mejorar su pronóstico, y la creación de mecanismos eficientes de atención que faciliten el paso de los pacientes por las diversas etapas de la atención.

Segundo, para nuestros pacientes, las creencias alrededor del origen, las causas y las características propias del cáncer, se convierten en elementos que permiten organizar los significados y la forma de afrontar y vivir la enfermedad. Los participantes mostraron tener conocimiento de la gravedad de la enfermedad desde antes del diagnóstico, basado en las experiencias de personas cercanas de su entorno familiar y social con un diagnóstico similar e información proporcionada por los medios de comunicación. La información giraba alrededor de un conocimiento compartido socialmente: la incurabilidad del cáncer, las diferencias según el órgano donde se inicia la enfermedad y la posibilidad de metástasis a otros órganos como indicador de progresión. Sin embargo, al momento del diagnóstico individual de los tres pacientes del estudio, la información sobre metástasis o del uso de quimioterapia paliativa no fue asociada con la posibilidad de muerte por enfermedad avanzada. Esto puede explicarse por la alta frecuencia con la que los pacientes atendidos por el grupo de cuidado paliativo y sus familias tienden a negar la condición de incurabilidad de la enfermedad y mantienen la esperanza de sanarse, a pesar de su deterioro físico. Esta falta de asociación entre el conocimiento previo de su enfermedad y su diagnóstico es un mecanismo de los participantes para defenderse de la amenaza que la enfermedad representa y poder responder adaptativamente a esta. El afrontamiento puede corresponder a la dimensión de evitación/negación del esquema de supervivencia de Moorey \& Greer (1998), que le permite al paciente proyectarse hacia el futuro y tener como expectativa la curación de la enfermedad, o al espíritu de lucha, en el cual el diagnóstico se convierte en un reto y se mantiene la esperanza de mantenerse asintomático.

Nuestros pacientes plantearon la herencia, el consumo de alcohol y tabaco, los alimentos contaminados, a Dios y al destino como el origen de 
la enfermedad. Sin embargo, no había una comprensión de la etiología multifactorial del cáncer, lo cual coincide con lo planteado en diversos estudios (Hanahan \& Weinberg, 2000; National Cancer Institute, 2012). Se observó dificultad para entender que una persona es susceptible de enfermar, aun sin tener los factores de riesgo conocidos para la enfermedad. La comprensión de las causas del cáncer es un factor que favorece la aceptación del diagnóstico y por esto se busca esta comprensión en los pacientes.

Tercero, las creencias construidas en relación con el pronóstico de la enfermedad fueron de relevancia para el afrontamiento adaptativo de la enfermedad y la adherencia al tratamiento. La percepción de amenaza que sintieron los pacientes en los primeros momentos desapareció cuando se controlaron los síntomas, evidencia de que hay una relación entre las molestias físicas y el bienestar emocional (González et al., 2006). Esto determina las creencias y significados de los pacientes sobre su enfermedad y su pronóstico, y favorece la adherencia al tratamiento y la confianza en el equipo médico (Soriano, 2002). Ante la falta de percepción de amenaza, el paciente en manejo con el grupo de cuidado paliativo no logra integrar la información sobre la incurabilidad de su enfermedad. En este periodo, cuando no hay presencia de síntomas, hay una vulnerabilidad emocional que requiere un acompañamiento para alcanzar bienestar psicológico, sin confrontar la defensa psíquica, que se considera adaptativa y que ha contribuido al afrontamiento de la enfermedad.

Estos pacientes reconocieron su diagnóstico pero no fueron conscientes de sus implicaciones. Esto es una negación de segundo orden, también encontrada en otros estudios (Doka \& Aldekoa, 2010), donde el paciente conoce sus síntomas y diagnóstico, pero rechaza su magnitud y ve los síntomas como hechos aislados y no como parte del proceso que puede llevar a la muerte. Esta dificultad para integrar la posibilidad de muerte coincide con lo señalado por Hurvitz (2009), quien plantea que las personas hacen representaciones de la enfermedad y le otorgan significados que se reflejan en sus reacciones y comportamientos. A pesar de ignorar la posibilidad de muerte, los participantes siguieron las recomendaciones del equipo médico. La negación cumplió una función protectora, y quizá adaptativa, pues no interfirió con la aceptación de la quimioterapia ni otros tratamientos de su enfermedad oncológica (Doka \& Aldekoa, 2010; Walsh, 2004).

Cuarto, las expresiones emocionales de los participantes variaron de acuerdo con el momento de la enfermedad que estaban viviendo. $\mathrm{Al}$ inicio, ante la falta de un diagnóstico claro y con los síntomas físicos, sentían mucha angustia, debido a la incertidumbre de no saber qué estaba sucediendo. Sus emociones fluctuaban entre la calma ante la posibilidad de un nuevo tratamiento y la ansiedad ante la reaparición o persistencia de los síntomas. Después de conocer su diagnóstico de cáncer y considerar la posibilidad de muerte, expresaron sorpresa, tristeza y angustia; esto es consistente con lo reportado en otros estudios (Die Trill \& López 2000; Malca, 2005). Una vez consideraron que su curación era una posibilidad debido a la disminución o desaparición de los síntomas, expresaron tranquilidad, bienestar y se proyectaron hacia el futuro, pensando en realizar actividades que hacían antes del diagnóstico.

Al iniciar el tratamiento y ante el control de los síntomas, desaparecieron las emociones negativas y disminuyó la tristeza, la sorpresa y la angustia, pues se desvaneció la posibilidad de muerte. Esto coincide con lo planteado por Lazarus (2000), quien refiere que la interpretación que la persona hace de la situación es lo que genera la reacción emocional. Cuando dejan de percibirla amenazante, logran estar tranquilos. Las respuestas emocionales de los pacientes en nuestro estudio fueron adaptativas, no asociadas con un trastorno mental. Esto se puede considerar un éxito del proceso de adaptación del paciente, como lo describen otros estudios (Rodríguez et al., 2007). Este afrontamiento les permitió 
integrar la experiencia y continuar con sus vidas, asumiendo los cambios generados por los tratamientos (cirugía, quimioterapia), asistiendo a las citas médicas, recibiendo quimioterapia, mientras seguían independientes en sus actividades básicas cotidianas y relacionándose con su familia.

Quinto, los recursos para la convivencia con su enfermedad les facilitan a los pacientes el proceso de afrontamiento que usualmente es desgastante fisica y emocionalmente. En nuestro estudio, los pacientes evidenciaron tres pilares de recursos: la confianza en Dios, los tratamientos médicos y el pensamiento positivo. Según Zabel (2000), las personas creyentes, ante el cáncer, reaccionan de tres maneras: abandonan el tratamiento porque Dios los va a sanar, sienten rabia con Dios y ven a Dios como un colaborador en su proceso de afrontamiento. En nuestro estudio, una fe en Dios fortalecida y una actitud positiva de forma permanente contribuyeron al éxito del tratamiento, aceptación de quimioterapia y tolerancia a sus efectos secundarios. La entrega de la enfermedad a Dios, como fue el caso de los tres participantes en este estudio, les dio paz y tranquilidad, disminuyó su carga emocional y les dio fortaleza para afrontar la situación que los agobiaba.

La familia fue también un recurso fundamental en el proceso de nuestros pacientes; el apoyo emocional a través de la compañía, la percepción de confianza, la seguridad económica y el respaldo en las actividades diarias fueron un apoyo fundamental para ellos. Muñoz, Espinosa, Portillo \& Rodríguez (2002) reportan que hay un buen ajuste de las familias al proceso de enfermedad cuando el paciente percibe apoyo y se siente reconfortado por sus seres queridos. La familia se moviliza, ajustándose a las demandas que surgen con el diagnóstico, se mantienen unidos y se adaptan para afrontar la condición de enfermedad (Hernández, 2009).

Las recomendaciones y los consejos de otros pacientes se convirtieron en otro recurso importante para nuestros pacientes. El aporte de otros elementos que abren posibilidades y alternativas $y$ el conocimiento de lo que les ha funcionado a otros les permiten aumentar su esperanza. Por esto, la creación de grupos de apoyo puede ser un espacio para facilitar el intercambio y la formación de redes entre los pacientes que asisten a recibir tratamientos oncológicos; esto puede resultar en una importante fuente de información y apoyo emocional durante el proceso de afrontamiento del cáncer.

Finalmente, existen unos obstáculos para el afrontamiento personal, familiar, social y de los aspectos propios de la enfermedad. En lo personal, los pensamientos centrados en la progresión de la enfermedad y las ideas asociadas ante la falta de futuro contribuyeron a la reducción de las actividades placenteras y al aislamiento social. El conocimiento de los participantes de que su enfermedad le generaba sufrimiento a la familia redujo la expresión de sus emociones. Así mismo, la familia evitó expresar sus sentimientos para no alterar al paciente en su proceso; esta situación puede generar que tanto el paciente como sus familiares sufran en silencio y no se abran espacios para conversar sobre lo que está sucediendo. Teniendo en cuenta que los significados se construyen en el lenguaje, $\mathrm{y}$ que este es el instrumento para configurar el mundo y la convivencia (Maturana, 1991), estos espacios conversacionales para el intercambio de emociones puede ser una estrategia de intervención terapéutica con el paciente y su familia.

El impacto negativo de la colostomía tanto física como emocionalmente limita las actividades simples de la vida cotidiana; resulta en cambios en la forma de vestir, las salidas recreativas, la alimentación, los cuidados básicos y la sexualidad (Martín \& Montesinos, 2010). La recuperación de la autonomía en las actividades de cuidado básico, como el aseo de su colostomía, es un factor importante ante este obstáculo. Esto coincide con lo reportado en otros estudios (Marquis et al., 2003; Wu et al., 2007), donde se muestra que hay una relación entre mejor calidad de vida y una mayor autoeficacia en estas actividades de autocuidado, que se recupera la intimidad en esta función 
corporal y se gana confianza en sí mismo. Las intervenciones de pareja con las personas afectadas con este diagnóstico y el manejo satisfactorio de su sexualidad y sus actividades sociales son parte integral del manejo de estos pacientes.

\section{Conclusiones}

La comprensión de las creencias, de las respuestas emocionales, de los recursos y de los principales obstáculos permitió profundizar en los significados construidos alrededor del cáncer.

Pese a que en el plano de lo cognitivo los participantes tenían conciencia de la asociación entre cáncer metastásico y posible muerte, el proceso emocional que enfrentaron hizo que ese conocimiento no pudiera asociarse con su propia condición. La aceptación de esta dura realidad lleva a que las personas le asignen significados ambivalentes a la enfermedad que oscilan entre el fin de los planes de vida y la posibilidad de recuperación completa y retorno a actividades previas. Estos significados permitieron el funcionamiento de los participantes de una manera adaptativa, pues fueron adherentes a los tratamientos y continuaron con sus actividades cotidianas, sin entregarse a la enfermedad y manteniendo un espíritu de lucha en la manera en que han asumido su proceso. Para estos tres participantes, sus significados estuvieron asociados a la recuperación de la salud, ellos no presentaban molestias físicas y lo registraron como mejoría que estaba conduciendo a la curación y no había percepción de muerte.

Una de las premisas que antecedieron a esta investigación fue que tener una enfermedad avanzada, progresiva e incurable se asociaba con la muerte. Esta premisa habla de la investigadora, de sus creencias, de su historia y de sus conocimientos acerca de los cuidados paliativos, sobre su definición y características. En la guías de la National Comprehensive Cancer Network (NCCN, 2012) se describe a la situación de la enfermedad terminal como fuente de sufrimiento y los resultados de esta investigación y los significados de estos tres participantes no coinciden con esto, ya que ellos no relacionaron su condición médica actual con la muerte.

Los significados asignados al cáncer dependieron de manera importante de la ausencia de síntomas y del proceso de elaboración emocional necesario para hacer frente a esta enfermedad. Para el caso de estos participantes, los significados estuvieron asociados con una condición crónica grave, no necesariamente conducente a la muerte, y cuyos tratamientos, aun cuando se realizan de manera paliativa, fueron considerados por los participantes como si estuvieran orientados hacia la curación. Estos significados podrían variar según la fase de la enfermedad en la que se encuentren las personas, puesto que cada una de ellas tiene unas características específicas y exigencias tanto físicas como emocionales. Así mismo, el afrontamiento se verá afectado, pues sus reacciones dependen de la percepción de amenaza que puedan sentir.

Otro aporte fundamental de esta investigación, en consonancia con los estudios en el tema, es el apoyo familiar como recurso para el afrontamiento de la enfermedad, pues se convierte en fuente de cuidados básicos. Los familiares asumen el cambio de roles para suplir las actividades cotidianas de los participantes, brindan acompañamiento y motivan a continuar adelante con el tratamiento. Las intervenciones que se hagan con la familia pueden ir encaminadas a proporcionar elementos que contribuyan al cuidado del cuidador y a generar recursos que faciliten el proceso de duelo anticipatorio cuando la enfermedad progrese.

Entre las limitaciones del estudio se identificó que el número de participantes fue reducido. Un mayor número de personas permitiría hacer una mirada más amplia sobre los significados del proceso de enfermedad y su afrontamiento. Otro aspecto limitante es que no se registraron las voces de la familia, y esta es importante para la construcción de significados del participante. Además, no se abordó la perspectiva de género. Finalmente, el 
contexto socioeconómico de los tres participantes fue el mismo, lo cual no dejó lugar a contrastar la información y buscar posibles diferencias.

Como resultado de este estudio, se pueden hacer algunas sugerencias para futuras investigaciones, por ejemplo, profundizar en los efectos psicológicos de la colostomía, puesto que esta puede ser fuente de preocupaciones para el paciente y llegar a generarle sufrimiento; podrían surgir de esta exploración elementos útiles para diseñar e implementar intervenciones grupales de tipo psicoeducativo que faciliten la adaptación a los cambios que genera la colostomía en las diferentes áreas de la vida cotidiana y promover espacios donde puedan construirse redes entre personas ostomizadas.

En relación con las implicaciones para la práctica clínica, es importante tener en cuenta el proceso de comunicación que se genera entre el paciente, la familia y el equipo médico. Además, la inclusión de la familia en las consultas e intervenciones terapéuticas es fundamental para identificar las necesidades del paciente, buscar su bienestar físico y emocional y privilegiar sus necesidades sobre las necesidades de la familia, que en ocasiones tienden a generar barreras que impiden que se dé un proceso de comunicación abierto con el paciente. Hay que comprender al paciente, escuchar sus voces y sus necesidades y partir de sus significados para llevar a cabo un trabajo donde se le reconozca en su individualidad para que, así, las intervenciones sean más ajustadas a su realidad y el acompañamiento más efectivo en su proceso de afrontamiento de la enfermedad.

Para los clínicos que trabajan en este campo queda una invitación a preguntarse acerca de sus significados sobre la enfermedad, la muerte, el cáncer y el sufrimiento, ya que en la interacción con esos seres humanos que padecen esta grave enfermedad surgen nuevos significados constantemente. No es posible estar en relación con otros sin involucrarse con ese otro. En el trabajo, en esta área es fundamental el trabajo con el ser del terapeuta, formularse preguntas de tipo existencial: ihe pensado en mi propia muerte?, ¿cómo visualizo mi muerte?, ¿he hablado de la muerte con mis seres queridos?, ¿saben ellos qué es para mí una muerte digna?, ¿conozco lo que desean ellos al final de sus días?, que le permitan tener una postura más clara frente al tema y reconocer las tensiones que se pueden crear en la interacción con los pacientes.

Se reitera la importancia de acompañar al otro desde su mundo de significados, darse el permiso de escucharlo con genuina curiosidad para poder así construir una relación de acompañamiento que permita a ese ser humano sentirse valorado, validado y amado.

\section{Referencias}

Anderson, H. \& Colapinto, J. (1999). Conversación, lenguaje y posibilidades: un enfoque posmoderno de la terapia. Madrid: Amorrortu.

Anderson, H. \& Goolishian, H. (1988). Human systems as linguistic systems: Evolving ideas about the implications for theory and practice. Family Process, 27, 371-393.

Ángel, L., Giraldo, A. \& Pardo, C. (2004). Mortalidad por cánceres del aparato digestivo en Colombia entre 19801 1998: análisis de tendencias y comparación regional. Revista Facultad de Medicina Universidad Nacional Colombia, 52 (1), 19-37.

Arcila, P., Mendoza, Y., Jaramillo, J., \& Cañón, O. (2010). Comprensión del significado desde Vygotsky, Bruner y Gergen. Diversitas: Perspectivas en Psicología (enero-junio), 37-49.

Bayés, R. (2006). Afrontando la vida, esperando la muerte. Madrid: Alianza.

Blair, L. \& Cardona, M. (2011). Pacientes con cáncer en fase terminal: una mirada fenomenológica existencial. International Journal of Psychological Research, 1(2), 13-20.

Broche-Pérez, Y. \& Medina, W. (2011). Resiliencia y afrontamiento: una visión desde la psicooncología. Documento procedente del $12^{\mathrm{a}}$ Congreso Virtual de Psiquiatría, Interpsiquis, Cuba. 
Die Trill, M. (2007). Impacto psicosocial del cáncer colorrectal. Psicooncología. Revista SEOM, 12(1), 19-21.

Die Trill, M., \& López, E. (2000). Aspectos psicológicos en cuidados paliativos: la comunicación con el enfermo y su familia. Madrid: ADES.

Doka, K. \& Aldekoa, J. (2010). Psicoterapia para enfermos en riesgo vital. Barcelona: Desclée de Brouwer.

Escalante, E. \& Páramo, M. (2011). El análisis de los datos en la investigación cualitativa. En E. Escalante \& M. Páramo (Eds.), Aproximación al análisis de datos cualitativos: aplicación de la práctica clínica (pp. 219-266). Mendoza: Universidad de Aconcagua.

Ferlay, J. (2010). Estimates of worldwide burden of cancer in 2008: GLOBOCAN 2008. International Journal of Cancer, 127(2), 893-971.

Gergen, K., \& Warhus, L. (s. f.). La terapia como una construcción social: dimensiones, deliberaciones, $y$ divergencias. Recuperado de: http://www. swarthmore.edu/Documents/faculty/gergen/ LA_TERAPIA.pdf

González, O., Fonseca, J., \& Jiménez, L. (2006). El cáncer como metáfora de muerte o como opción para resignificar la vida: narrativas en la construcción de la experiencia familiar y su relación con el afrontamiento del cáncer de un hijo menor de edad. Revista Diversitas Perspectivas en Psicología, 2(2), 259-277.

Hanahan, D. \& Weinberg, R. (2000). The halmarks of cancer. Cell, 100, 57-70.

Hernández, A. (2009). Familia, ciclo vital y psicoterapia sistémica breve. Bogotá: El Búho.

Hernández, A. (2010). Vinculos, individuación y ecología humana: hacia una psicología clínica compleja. Bogotá: Universidad Santo Tomas de Aquino.

Hurvitz, M. (2009). Las representaciones sociales que tienen los pacientes y médicos de los signos y síntomas del cáncer colorrectal. Revista de la Asociación de Coloproctología del Sur, 4(1), 1-95.
Labianca, R., \& Merelli. B. (2010). Screening and diagnosis for colorectal cancer: Present and future. Tumori, 96(6), 889-901.

Lazarus, R. (2000). Estrés y emoción: manejo e implicaciones en nuestra salud. Bilbao: Desclee de Brouwer.

McVey, J., Madill, A., \& Fielding, D. (2001). The relevance of lowered personal control for patients who have stoma surgery to treat cancer. British Journal of Clinical Psychology, 40(4), 337-360.

Malca, B. (2005). Psicooncología: abordaje psicoemocional en oncología. Persona y Bioética, 9(2), 64-67.

Martín, B., \& Montesinos, A. (2010). Ayuda emocional entre pacientes ostomizados. Biblioteca Las Casas, 7(2), 1-33. Recuperado de: http://www. index-f.com/lascasas/documentos/lc0600.php

Martín, B., Panduro, R., Crespillo, Rojas, L., \& González, S. (2010). El proceso de afrontamiento en personas recientemente ostomizadas. Index de Enfermería, 19(2), 115-119.

Martín, D., Zanier, J., \& García, F. (2003). Afrontamiento y calidad de vida: un estudio de pacientes con cáncer. Psico-USF, 8(2), 175-182.

Marquis, P., Marrel, A., \& Jambon, B. (2003). Quality life in patients with stomas: The Montreux study. Ostomy Wound Management, 49(2), 48-55.

Maturana, H. (1991). El sentido de lo humano. Hachette/Comunicación, 11, 34-35.

Muñoz, F., Espinosa, J. M., Portillo, J., \& Rodríguez G. (2002). La familia en la enfermedad terminal. Medicina de Familia, 3(4), 262-268.

National Cancer Institut. (2012). Mitos y creencias sobre el cáncer. Recuperado de: http://www. cancer.gov/espanol/cancer/mitos-creencias

Oliveros, E., Barrera, M., Martínez, S. y Pinto, T. (2010). Afrontamiento ante el diagnóstico de cáncer. Revista de Psicología GEPU, 1(2), 19-29.

Pascual, A. (2003). Cuidados paliativos. Humanitas, Humanidades Médicas, 1(1), 85-92.

Piñeros, M., Pardo, C., Gamboa, O. \& Hernández, G. (2010). Atlas de mortalidad por cáncer en Colombia. Bogotá: Instituto Nacional de 
Cancerología, Ministerio de la Protección Social de Colombia.

Quintana, A. (2006). Metodología de investigación científica cualitativa. En A. Quintana \& W. Montgomery (Eds.), Psicología: tópicos de actualidad (pp. 47-84). Lima: UNMSM.

Rodríguez, B., Bayón, C., Orgaz, P., Torres, G., Mora, F. \& Castelo, B. (2007). Adaptación individual y depresión en una muestra de pacientes oncológicos. Psicooncología, 4(1), 7-19.

Rojas-May, G. (2006). Estrategias de intervención psicológica en pacientes con cáncer de mama. Revista Médica Clínica Las Condes, 17(4), 194-197.

Sandoval, C. (2002). Enfoques y modalidades de investigación cualitativa: rasgos básicos. En Investigación cualitativa (pp. 53-95). Bogotá: ICFES.

Silva, A. L. \& Shimizu, H. E. (2006). El significado del cambio en el modo de vivir de la persona con colostomía intestinal definitiva. Revista Latinoamericana de Enfermería, 14(4), 483490. Recuperado de http://www.scielo.br/scielo. php?pid=S0104-11692006000400003\&scrip$\mathrm{t}=$ sci_abstract\&tlng $=\mathrm{es}$

Sociedad Española de Cuidados Paliativos (SECPAL). (s. f.). Guía de cuidados paliativos. Madrid: autor. Recuperado de http://www.secpal.com/ index.php

Soriano, J. (2002). Reflexiones sobre el concepto de afrontamiento en psicooncología. Boletín de Psicología, 75, 73-85.
Supersalud (2009). Sistema de indicadores de alerta temprana. Circular Externa (56).

Taylor, S. J. \& Bogdan, R. (1990). Introducción a los métodos cualitativos. Barcelona: Paidós.

Tarragona, M. (2006). Las terapias posmodernas: una breve introducción a la terapia colaborativa, la terapia narrativa y la terapia centrada en soluciones. Psicología Conductual, 14(3), 511-532.

Valentín, V., Murillo, M., Valentín, M. \& Pascual, R. (2003). Modelo de organización de los cuidados paliativos. En V. Valentín (Ed.), Oncología en atención primaria (pp. 747-770). Madrid: Nova Sidonia Oncología y Hematología.

Vinaccia, L., \& Arango, C. (2003). Evaluación de la calidad de vida y su relación con la cognición hacia la enfermedad en pacientes colostomizados con diagnóstico de cáncer colorrectal. Suma Psicológica, 10(1), 43-65.

Walsh, F. (2004). Procesos familiares fundamentales para la resiliencia. En Resiliencia familiar: estrategias para su fortalecimiento (pp. 79-122). Buenos Aires: Amorrortu.

Wenk, R. (2007). Manual de cuidado paliativo. Buenos Aires: Fundacion Femeba-Programa Argentino de Medicina Paliativa.

Wu, H., Chau, J. \& Twinn, S. (2007). Efficacy and quality of life among stoma patients in Honk Kong. Cancer Nursing, 30(3), 186-193.

Zabel, C. (2000). Cáncer y creencias espirituales. Boletín de Psicología, 2(5).

\section{Fecha de recepción: 14 de julio de 2014 fecha de aceptación: 17 de diciembre de 2014}

